$16^{\text {th }}$ International Congress of Metrology, 07004 (2013)

DOI: $10.1051 /$ metrology/201307004

(c) Owned by the authors, published by EDP Sciences, 2013

\title{
Evaluation of the beta spectral shapes
}

\author{
Charlène Bisch ${ }^{1}$, Xavier Mougeot ${ }^{1, a}$ and Marie-Martine Bé ${ }^{1}$ \\ ${ }^{1}$ CEA, LIST, Laboratoire National Henri Becquerel (LNE-LNHB), Gif-sur-Yvette, F-91191, France
}

\begin{abstract}
Following an increased demand from users for a precise knowledge of beta spectra coupled with well-established uncertainties, LNHB is leading a study of beta spectral shapes at the metrological level. The evaluation of these beta spectra, especially to reach metrological uncertainties, requires new measurements and new calculations with high precision. The first part of this contribution will describe a new experimental setup for the measurement of beta energy spectra based on a silicon detector with a very thin entrance window and operating at liquid nitrogen temperatures under high-vacuum. Monte Carlo simulations were performed to optimize the detection chamber and source holder. Some preliminary results are included. The second part summarizes the calculations already implemented. These complex calculations are compared to some measurements of beta spectra obtained by using metallic magnetic calorimeters, and highlight the major contribution of the atomic screening and exchange effects at low energy.
\end{abstract}

\section{Introduction}

The study of the shape of beta spectra is experiencing a resurgence of interest having been little studied since the late 1970s. At that time, these spectra were thought to be known accurately enough. However, at present, precise knowledge of the shape of energy spectra, coupled with well-established uncertainties, are sought by users from the nuclear power industry, the medical care sector or for ionizing radiation metrology.

In ionizing radiation metrology, the energy spectra of beta decays are often needed, especially when measurements are carried out using Liquid Scintillation Counting. The modelling of light emission used in the Triple to Double Coincidence Ratio (TDCR) method, to establish a relationship between the detection efficiency and the experimental TDCR ratio, requires the knowledge of the shape of the beta spectra for beta-decaying nuclides [1]. Due to the threshold effect, the reliability of the TDCR-Cerenkov technique is even more sensitive to the accuracy of the shape of beta spectra [2].

Moreover, biologically targeted radiotherapy using new radionuclides is developing as a means of cancer treatment, along with the production of new radiopharmaceuticals [3]. As in any treatment using ionizing radiation, the knowledge of the dose delivered within the tumour, and to the rest of the body, is essential to ensure the efficacy and the safety of the treatment. One of the problems is related to the calculation of the dose delivered in the volumes of interest according to the quantity of radionuclide which is present there. The precise knowledge of the shapes of beta spectra, particularly in the low-energy part of the spectrum, is essential for calculating the dose deposited at the level of biological cells [4]. Indeed, the very low energy electrons have a high contribution to the dose because the linear energy transfer of electrons greatly increases as their kinetic energy decreases [5].

As the primary laboratory for measurements of radioactivity in France, the LNHB requires a computer code to meet the demands of users, which should be validated against experiment. Hence, the evaluation of the beta spectral shapes leads us to be interested in both experimental and theoretical studies. First, the operational device using a semiconductor $\mathrm{Si}$ detector (easy to implement, linear response function, good energy resolution) will be described, which has been developed in order to measure spectral shapes and to quantify uncertainties. This is the aim of a current $\mathrm{PhD}$ programme and is embedded in the first Work Package of the 2011 European Metrology Research Programme "Metrology for Molecular Radiotherapy". Then, our current theoretical abilities will be summarized.

\section{Experimental device}

\subsection{Requirements}

The main difficulties in beta spectrometry arise because the spectra may be distorted by the detection system. Specific requirements are necessary to limit the sources of deformation and to maximize the signal-to-noise ratio [6]. Silicon detectors are operable at room temperature,

\footnotetext{
$\bar{a}$ Corresponding author: xavier.mougeot@cea.fr
} 
however, thermally-generated leakage currents contribute significantly to the noise level. Operation at liquid nitrogen temperature offers marked improvements in resolution and the signal-to-noise ratio, especially for low energies. Operation at low temperature, in turn, dictates a further design criterion: the detector must be kept under vacuum to prevent atmospheric moisture from condensing and freezing on the detector's surface [7]. However, the vacuum pump induces microphonics which have to be attenuated. Furthermore, physical phenomena like self-absorption in the source, detector dead zones, angle of incidence, scattering and backscattering or bremsstrahlung also have a great influence on the measured spectral shape. A high-vacuum provides another advantage, namely a low scattering environment. However, we must also choose carefully the materials and define the geometry of the detection chamber and source holder. Finally, for each radionuclide, a chemical study must be undertaken to determine the best method for preparing thin and homogeneous sources [8].

\subsection{Description}

The detection chamber is a cube milled on its six faces. Clearly the bigger the cube, the lower the scattering, but we must also avoid a cumbersome system. The best trade-off found by Monte Carlo simulations using Geant4 [9] is a cube with $170 \mathrm{~mm}$ sides made of aluminum. A schematic view is shown in figure 1.

Figure 1. Schematic view of the beta spectrometer.

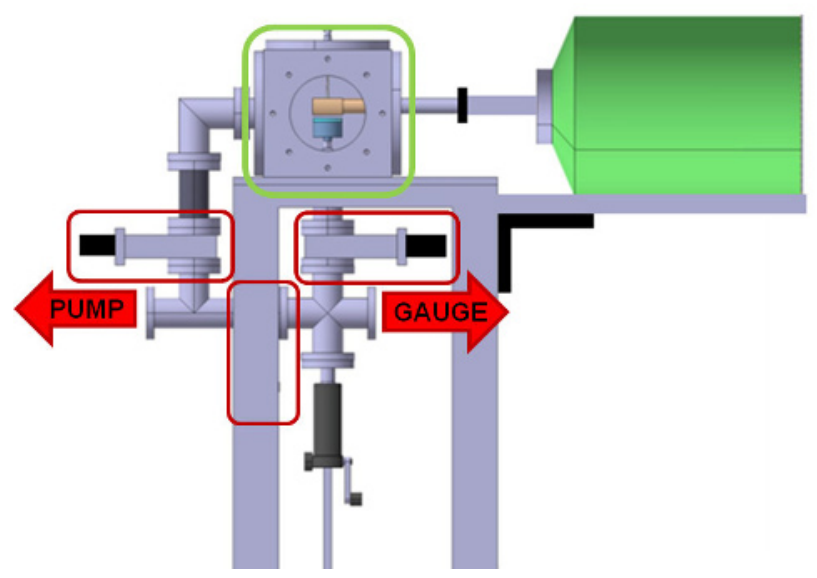

We want to avoid, as far as possible, the detection of backscattered electrons coming from the source holder. Backscattering is more pronounced for electrons of low energy and absorbers with high atomic number [10]. Geant4 simulations were conducted from the Geant 4 benchmark TestEm5 adapted to our application. We tested two materials for the source holder: aluminium and PEEK (polyether ether ketone) which is an organic polymer thermoplastic. PEEK is the best plastic to use for vacuum applications because it does not outgas. A $500 \mathrm{keV}$ monoenergetic beam was simulated, impinging on a full block of material, and then, conserving the same geometry, with a $1 \mathrm{~cm}$ recess milled into the block. Finally, the geometry adopted for the source holder is shown in figure 2 . The notches on the side allow pressure equalization across the source.

Figure 2. View of the source holder.

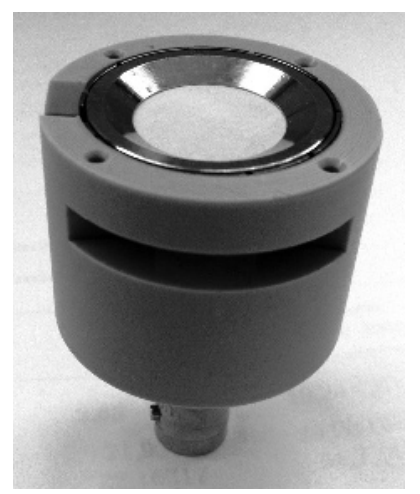

The detector is a PIPS (Passivated Implanted Planar Silicon) manufactured by Canberra. The main advantages of this technology for our application are a very thin entrance window of $50 \mathrm{~nm} \mathrm{Si}$ equivalent and a low leakage current. The detector is $500 \mu \mathrm{m}$ thick and has an active surface area of $450 \mathrm{~mm}^{2}$. The electronics system is a classical spectrometry chain, using an Amptek "CoolFET" charge sensitive preamplifier cooled by the Peltier effect.

\subsection{Preliminary results}

\subsection{1 ${ }^{109} \mathrm{Cd}$ calibration source}

As an initial test we have measured the spectrum of a ${ }^{109} \mathrm{Cd}$ source, which emits monoenergetic conversion electrons (Table 1) that appear as sharp peaks in the spectrum (figure 3). To determine the influence of temperature on the detector, two spectra were acquired with and without liquid nitrogen cooling. As expected, the energy resolution is better at $77 \mathrm{~K}$ than $300 \mathrm{~K}$. These spectra are preliminary because the electronics were not optimized during acquisition, hence, the resolution may improve. The response function for this energy range seems to be linear. Monte Carlo calculations are still in progress in order to complete the analysis.

Table 1. Characteristic electron emissions from ${ }^{109} \mathrm{Ag}$ following the decay of ${ }^{109} \mathrm{Cd}$. The data are from [11].

\begin{tabular}{|c|c|c|}
\hline Energy (keV) & Intensity (\%) & Type \\
\hline 21.7 & 20.6 & Auger K \\
\hline 62.52 & 40.8 & ec K \\
\hline 84.46 & 44.8 & ec L \\
\hline 87.67 & 9.28 & ec M \\
\hline
\end{tabular}


Figure 3. ${ }^{109} \mathrm{Cd}$ conversion electron spectrum. The $85 \mathrm{keV}$ peak is a mixture of the $\mathrm{L}$ and $\mathrm{M}$ conversion electrons (Table 1).

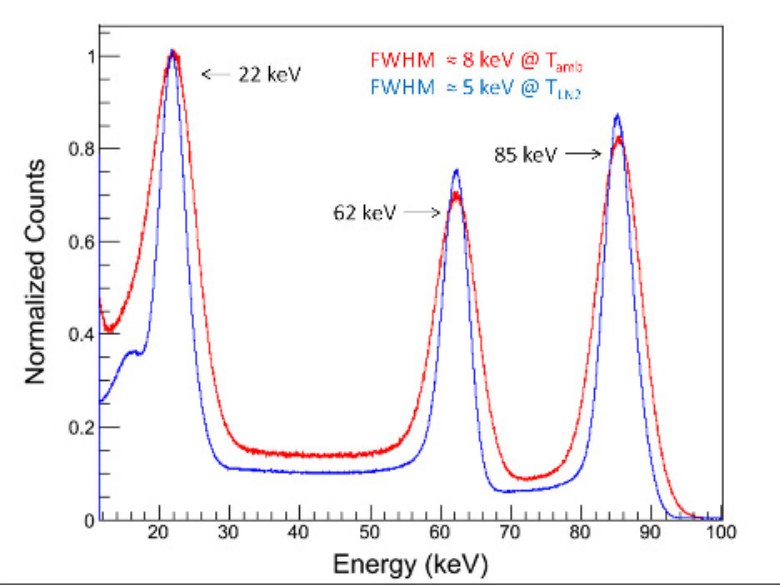

\section{3. $2{ }^{36} \mathrm{Cl}$ beta spectrum}

A preliminary measurement of a ${ }^{36} \mathrm{Cl}$ source was carried out. As expected, the high energy electrons that pass through our $500 \mu \mathrm{m}$ thick detector induce a folding of the spectrum. The realistic geometry of the experimental setup was implemented in our simulations. We used the experimental shape factor extracted from the measurement of Rotzinger [12] for the primary emitted beta spectrum. The data were adjusted using an approximate linear energy calibration. As shown in figure 4 , our simulation reproduces well the main distortion of the measured spectrum. A complete and careful study of the detector response function will provide a precise energy calibration and should improve the agreement with our simulation.

Figure 4. Comparison between the ${ }^{36} \mathrm{Cl}$ measured and simulated beta spectra.

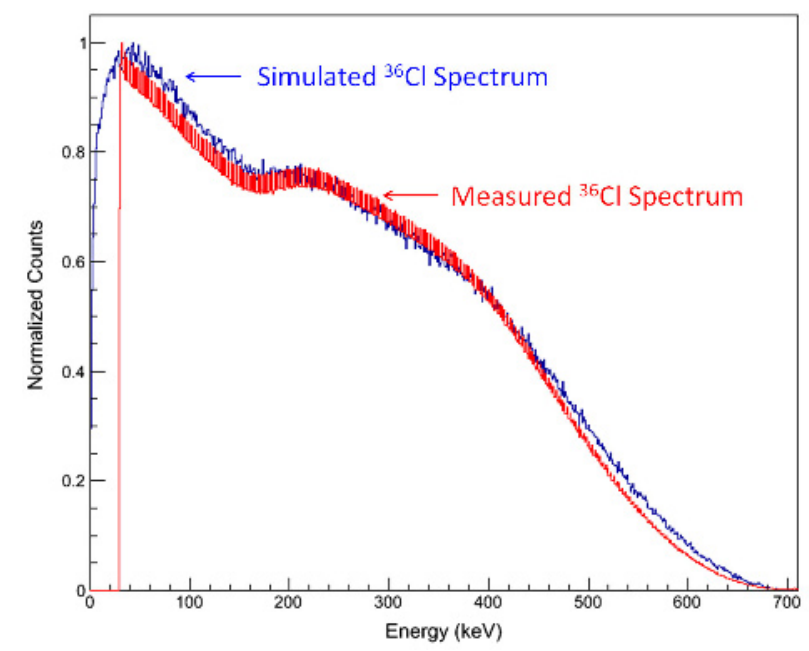

\section{Calculation of beta spectra}

\subsection{Allowed and forbidden unique transitions}

During the beta decay of a radionuclide, the available energy is split between the electron, the neutrino and the daughter nucleus. This three-body decay implies a continuous energy spectrum for electrons. The shape of this spectrum is also influenced by the nature of the transition that can be "allowed" or "forbidden" depending on the structure of the nuclei involved, and by certain atomic effects. Electrons thus have continuous energy spectra of varied shapes.

A beta spectrum is the product of a weak interaction coupling constant, a statistical phase space factor, the socalled Fermi function which corrects for the Coulomb effects, and a shape factor. The phase space factor simply reflects the momentum distribution between the electron and the neutrino. The shape factor contains all the remaining energy dependencies, such as leptonic and nuclear matrix elements or corrections for atomic effects.

A computer code, BetaShape, in development at LNHB to calculate the beta spectra for allowed and forbidden unique transitions has already been described elsewhere [13].

\subsection{Atomic effects}

Atomic effects play an important role in determining the shape of beta spectra, mainly at low energy. The sudden change of the nuclear charge can induce atomic excitations or internal ionizations, which are respectively called shake-up and shake-off effects and are not expected to have a contribution higher than $0.1 \%$. The two major atomic effects are screening and the exchange effect.

\subsubsection{Screening}

Atomic electrons partially screen the nuclear charge seen by the beta electron: this is the well-known screening effect.

When calculating a beta spectrum, the screening effect is generally corrected for using a constant ThomasFermi potential which is subtracted from the total energy of the particle [14]. This method creates a non-physical discontinuity at the minimum energy defined by the potential [13]. This minimum energy is $\leq 20 \mathrm{keV}$, hence the discontinuity does not generally affect the practical use of the spectrum. Physically, the influence of the atomic electrons is only expected to be significant when the beta wavelength is comparable to the atom size, thus at low energy.

As it has been shown in [15], this simple method, which allows analytical wave functions to be used, cannot be reasonable for high $\mathrm{Z}$ and at low energy. The usual way is to correct the Coulomb potential for the influence of the atomic electrons. Such screened potentials can be found in [16]. However, the Dirac equations for the wave functions can no longer be solved analytically, hence we have followed the numerical method described in detail in [17].

Beta spectra are classically calculated using the electron radial wave functions evaluated at the nuclear radius. To take the screening into account, it is not just sufficient to renormalize the wave functions and evaluate them at the nuclear radius because the screened potentials 
are very weak at this distance, and so, the modification is completely negligible over the entire range of the spectrum. It is, in fact, necessary to take into account the spatial extension of the wave functions. As described in [17], this can be done by evaluating the transition matrix of the corresponding beta-decay. However the calculations become very complicated because the electron wave functions are coupled with the nuclear matrix elements involved. To avoid this difficulty, we have defined a new, simple type of screening correction available for allowed and forbidden unique transitions [18].

\subsubsection{Exchange effect}

The exchange effect is also an atomic effect. It arises from the creation of a beta electron in a bound orbital of the daughter atom corresponding to one which was occupied in the parent atom. An atomic electron from the bound orbital simultaneously makes a transition to a continuum orbital of the daughter atom. This process cannot be distinguished from the direct decay to a final state containing one continuum electron.

Formalism of the exchange effect and consecutive calculations have already been set out in detail in [15] for allowed transitions. Indeed in this case, only the $n s$ orbitals are reachable by the beta electron, which greatly simplifies the calculations.

Harston and Pyper [19] pointed out that the screening must distort the wave functions and contribute significantly to their overlap. They took this effect into account using effective nuclear charges for the calculations of the wave functions involved. This does not concern the wave functions evaluated at the nuclear radius, for which the full nuclear charge has to be used. Following [19], the effective nuclear charges were determined from the mean radii of the orbitals, which were taken from [20] and [21] in the non-relativistic case (Hartree-Fock calculations), and from [22] in the relativistic case (Dirac-Fock calculations).

Finally, analytical electron radial wave functions were taken from [23] and are expressed in terms of confluent hypergeometric functions. Achieving the required accuracy on the overlap means evaluating this special function for complex arguments of large magnitudes.

\subsection{Results}

The beta spectra of ${ }^{63} \mathrm{Ni}$ [24] and ${ }^{241} \mathrm{Pu}$ [25] were recently measured using metallic magnetic calorimeters. Each source was enclosed in a gold absorber assuring a $4 \pi$ solid angle and $100 \%$ detection efficiency.

The radionuclide ${ }^{63} \mathrm{Ni}$ decays entirely by beta minus emission to the ground state of ${ }^{63} \mathrm{Cu}$. This allowed transitions has a maximum energy of $66.98 \mathrm{keV}$ [26].

The radionuclide ${ }^{241} \mathrm{Pu}$ decays mainly by beta minus emission to the ground state of ${ }^{241} \mathrm{Am}$. The nature of the transition is first forbidden non-unique, with a maximum energy of $20.8 \mathrm{keV}$ [27]. This transition fulfills the assumption of the $\xi$ approximation and can be calculated as allowed [15].
The low maximum energies of these two transitions make them case studies for evaluating the influence of atomic effects. These spectra were first compared to classical beta calculations, and then taking into account the screening and exchange effects. Our calculations are compared with the measured spectra in figure 5 for ${ }^{63} \mathrm{Ni}$ decay and in figure 6 for ${ }^{241} \mathrm{Pu}$ decay.

Figure 5. Comparison between the measured beta spectrum of ${ }^{63} \mathrm{Ni}$ from [24] and three calculated spectra: a classical allowed transition without screening or exchange, the same including screening, and a complete one including screening and exchange corrections.

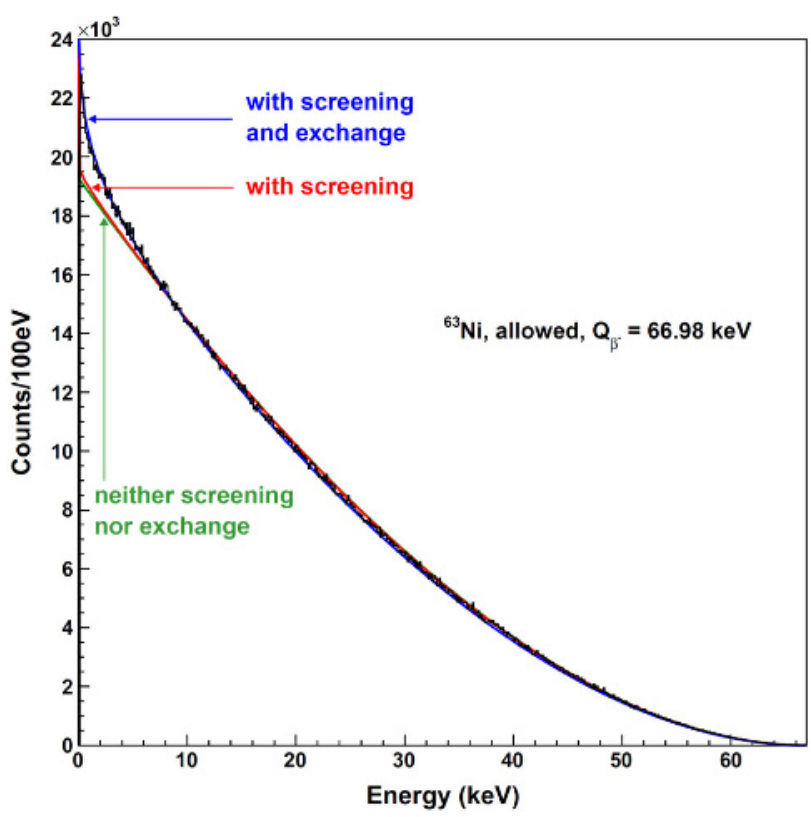

Figure 6. Comparison between the measured beta spectrum of ${ }^{241} \mathrm{Pu}$ from [25] and three calculated spectra: a classical allowed transition without screening or exchange, the same including screening, and a complete one including screening and exchange corrections.

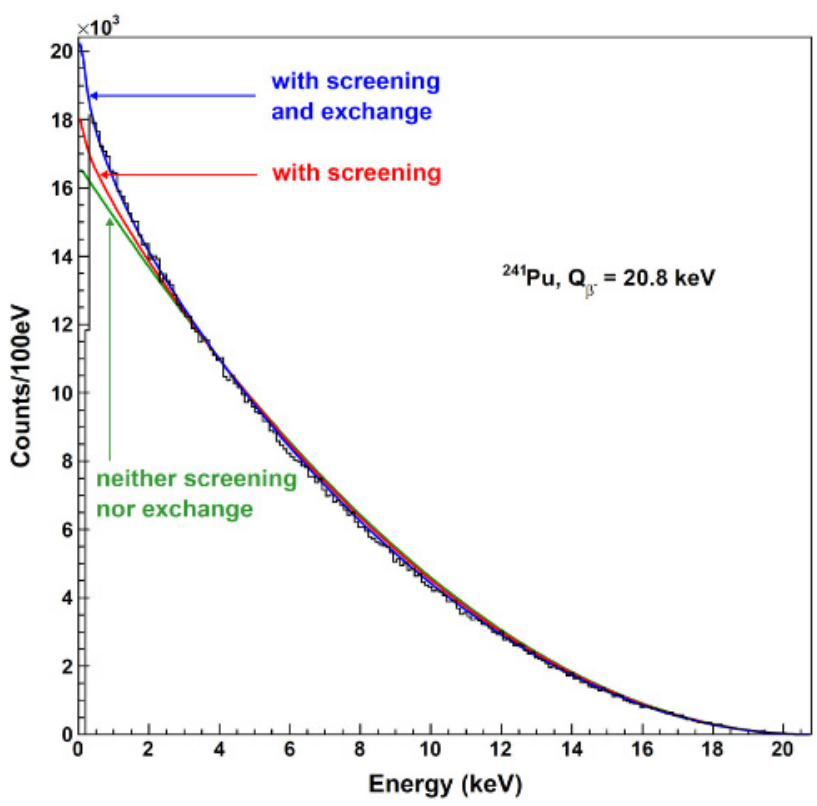

An excellent agreement over the entire energy range of the spectrum has been obtained in both decays, which validates our calculations of the influence of the atomic 
effects on beta spectral shapes. For ${ }^{63} \mathrm{Ni}$, taking the exchange effect into account leads to a mean energy lower by about $2.6 \%$. For ${ }^{241} \mathrm{Pu}$, the mean energy of the beta spectrum is lower by about $3.5 \%$ if the screening and the exchange effect are included in the calculations.

\section{Conclusion}

Our experimental setup is finalized and initial measurements have been carried out with success. Some distortion persists, but owing to the important development and simulation work, we should be able to characterize and quantify them with our Monte Carlo simulations. The next step will be to precisely study the response function of the detector in order to determine an experimental distortion function. Then we will carry out a series of measurements of well-known spectra to validate our method. This particular setup is well suited for measuring beta spectra with a maximum energy of about $1 \mathrm{MeV}$. It is also easily adjustable to a $5 \mathrm{~mm}$ thick $\mathrm{Si}(\mathrm{Li})$ detector, for measuring beta spectra up to about $3.5 \mathrm{MeV}$. We will then focus on unique and non-unique forbidden beta spectra to validate our code BetaShape.

Beta spectra of ${ }^{63} \mathrm{Ni}$ and ${ }^{241} \mathrm{Pu}$ are reproduced well in our calculations down to very low energies. The exchange effect is demonstrated to have a great influence on the spectrum shape within this energy range. As expected, the influence of the screening is weak for the low $\mathrm{Z}$ nuclide ${ }^{63} \mathrm{Ni}$ but has a significant contribution, equivalent to the magnitude of the exchange effect, for the high $\mathrm{Z}$ nuclide ${ }^{241} \mathrm{Pu}$. The bound and continuum wave functions involved in the exchange effect were evaluated with analytical expressions using effective nuclear charges. These effective charges were not determined at this stage with the same screened potentials used for the calculation of the continuum wave functions involved in the screening correction. We are now implementing a new code to calculate the exchange effect with wave functions identical to those used for the screening.

\section{References}

1. R. Broda, P. Cassette, K. Kossert, Metrologia 44, S36-S52 (2007)

2. C. Bobin et al., Appl. Radiat. Isot. 68, 2366 (2010)

3. C. Andrew Boswell, M.W. Brechbiel, Nucl. Med. Biol. 34, 757 (2007)

4. M. Bardiès, J.-F. Chatal, Phys. Med. Biol. 39, 961 (1994)

5. A.I. Kassis, Int. J. Radiat. Biol. 80, 789 (2004)

6. G. Grégoires, Nucl. Instr. Meth. 28, 346 (1964)

7. C.T. Prevo et al., Nucl. Instr. Meth. 55, 173 (1967)

8. W. Parker et al., Nucl. Instr. Meth. 7, 22 (1960)

9. S. Agostinelli et al., Nucl. Instr. Meth. Phys. Res. A 506, 250 (2003)

10. G.F. Knoll, Radiation Detection and Measurement, $2^{\text {nd }}$ edition, p.47 (1989)

11. M.-M. Bé et al., Table of Radionuclides Monographie BIPM-5 Vol. 1, edited by BIPM, Pavillon de Breteuil F - 92312 Sèvres, p.191 (2004)
12. H. Rotzinger et al., J. of Low Temp. Phys. 151, p.1087 (2008)

13. X. Mougeot et al., Proceedings of the LSC2010 International Conference, edited by $\mathrm{P}$. Cassette, University of Arizona, p.249 (2010)

14. R.H. Good, Phys. Rev. 94, 931 (1954)

15. X. Mougeot et al., Phys. Rev. A 86, 042506 (2012)

16. F. Salvat et al., Phys. Rev. A 36, 467 (1987)

17. H. Behrens, W. Bühring, Electron Radial Wave functions and Nuclear Beta Decay, Oxford Science Publications (1982)

18. X. Mougeot et al., Proceedings of International Conference on Nuclear Data for Science and Technology (ND2013), Nucl. Data Sheets, (to be published)

19. M.R. Harston, N.C. Pyper, Phys. Rev. A 45, 6282 (1992)

20. C.F. Fischer, The Hartree-Fock method for atoms, Wiley and Sons (1977)

21. J.B. Mann, At. Data Nucl. Data Tables 12, 1 (1973)

22. J.P. Desclaux, At. Data Nucl. Data Tables 12, 311 (1973)

23. M.E. Rose, Relativistic electron theory, Wiley and Sons (1961)

24. C. Le-Bret, PhD thesis, Université Paris 11 (2012)

25. M. Loidl et al., Appl. Radiat. Isot. 68, 1460 (2010)

26. M.-M. Bé et al., Table of Radionuclides Monographie BIPM-5 Vol. 3, edited by CEA/LISTLNHB, BIPM, p.29 (2006)

27. M.-M. Bé et al., Table of Radionuclides Monographie BIPM-5 Vol. 4, edited by CEA/LISTLNHB, BIPM, p.259 (2008) 